\title{
Purpuricenus Wachanrui Levrat und seine Varietäten.
}

Bei dieser schönen persischen Art ist das Halsschild roth mit schwarzen Seitenrändern, die schwarze Farbe tritt am Vorderrand mit 2, am Hinterrand mit 3 schwarzen Zacken in die rothe Farbe ein, der mittlere Hinterrandszacken verlängert sich nach vorn bis über die Mitte; bei einem meiner Stücke aus Malatia sind die Zacken kaum angedeutet. Auf den Flügeldecken befindet sich auf der Mitte eine gemeinsame breite rothe Binde, die durch die schwarze Naht mehr oder weniger getrennt ist, aufsen ist sie am breitesten und reicht vorn bis fast an die Schulter, an der Naht vorn schief nach hinten, hinten schief nach vorn begrenzt, so dafs sie an der Naht am schmälsten ist. Am Ende der rothen Binde befindet sich eine schwarze Sammetbinde. Hierher rechne ich als Stammart den von Levrat beschriebenen Wachanrui, sowie den von Witte, Berl. Ent. Z. 1871, pag. 207, beschriebenen P. Hausknechti aus Kurdistan.

Bei der ebenda pag. 208 bekannt gegebenen var. aleppensis Witte hat die schwarze Farbe des Halsschildes so überhand genommen, dafs nur 2 oblonge rothe Flecke übrig bleiben, auf der Naht sind die beiden schwarzen Hälften deutlich mit einander verbunden. - Von Aleppo.

Var. bilunatus Schaufs., Nunq. ot. 1871, pag. 210, hat ganz rothes Halsschild und die rothe Deckenmakel ist an der Naht breit unterbrochen, innen mehr abgerundet. - Von Cypern.

Ob Nicocles Schaufs., l. c. 209, hierher zu ziehen ist, weifs ich nicht, der Thorax wird ochraceus genannt, disco bipunctato, basi triplagiato; elytris pallidis, disperse punctulatis (während alle bis jetzt erwähnte Formen antice profunde rugoso-punctati sind), basi nigris, disco plaga minuta transversa punctoque post medium nigris. Die Behaarung wird weifslich genannt. - Von Cypern.

Von Herrn Oberst-Lieutenant von Schönfeldt in Weimar erhielt ich eine neue Form, die:

Var. Schönfeldti Heyden aus Sultanabad in Persien. Bei dieser Varietät ist die rothe Farbe ganz geschwunden, sie ist ganz schwarz und macht dadurch einen besonderen Eindruck. Dafs aber auch hier die rothe Farbe nur verdrängt ist, beweist ein mit unbewaffnetem Auge kaum sichtbares blutrothes, stecknadelgrofses Fleckchen am Deckenaufsenrand im ersten Drittel. Herr von Schönfeldt besitzt ein $\mathbf{z w e i t e s}$ ebenso gezeichnetes Stück; das meinige ist ein 오 von 15 mill. Länge, während meine typischen Amasia-Stücke nur 12 mill. lang sind. Die schwarze Tomentbinde der Decken ist sehr deutlich.

Major z. D. Dr. L. von Heyden. 


\section{$2 \mathrm{BHL}$ Biodiversity Heritage Library}

Heyden, Lucas von. 1890. "Purpuricenus Wachanrui Levrat und seine Varietäten." Deutsche entomologische Zeitschrift 1890(3), 79-79. https://doi.org/10.1002/mmnd.48018900310.

View This Item Online: https://www.biodiversitylibrary.org/item/103589 DOI: https://doi.org/10.1002/mmnd.48018900310

Permalink: https://www.biodiversitylibrary.org/partpdf/235473

\section{Holding Institution}

Harvard University, Museum of Comparative Zoology, Ernst Mayr Library

\section{Sponsored by}

Harvard University, Museum of Comparative Zoology, Ernst Mayr Library

\section{Copyright \& Reuse}

Copyright Status: Public domain. The BHL considers that this work is no longer under copyright protection.

This document was created from content at the Biodiversity Heritage Library, the world's largest open access digital library for biodiversity literature and archives. Visit BHL at https://www.biodiversitylibrary.org. 